\title{
THE OXYGEN-RICH SUPERNOVA REMNANT IN \\ THE SMALL MAGELLANIC CLOUD
}

\author{
William P. Blair \\ The Johns Hopkins University, Baltimore, Md., U.S.A. \\ John C. Raymond \\ Harvard-Smithsonian Center for Astrophysics, Cambridge, Ma., U.S.A. \\ John Danziger and Francesca Matteucci \\ European Southern Observatory, Garching bei Munchen, West Germany
}

\begin{abstract}
We report ultraviolet and optical spectra of 1E 0102-7219, the oxygen-rich supernova remnant in the Small Magellanic Cloud. The UV data contain strong lines of oxygen, carbon, neon, and magnesium. OI recombination lines in the optical and UV permit the relative line intensities to be determined from $1200 \AA$ to 1 micron. Models assuming shock excitation and X-ray photoionization have been calculated and compared with the observations.
\end{abstract}

\section{Introduction}

The supernova remnant $1 \mathrm{E}$ 0102-7219 was first identified in the Einstein X-ray survey of the Small Magellanic Cloud (SMC) by Seward and Mitchell (1981). Measurements with the Imaging Proportional Counter on Einstein showed the object to be the second brightest $\mathrm{X}$-ray source in the SMC and to have a relatively soft X-ray spectrum, similar to SNRs in our Galaxy and the Large Magellanic Cloud (LMC). However, the IPC count rate was $\geq 10$ times that of the next brightest SMC remnant.

Optically, Dopita, Tuohy and Mathewson (1981) identified the remnant with a faint ring of filaments $\sim 24$ arcsec in diameter visible only in the light of $[\mathrm{O} I I I] \lambda 5007$, adjacent to the $\mathrm{H}$ II region N76A. At a distance of $59 \mathrm{kpc}$ for the SMC, the diameter of the $[\mathrm{O} \mathrm{III]} \mathrm{ring}$ is $6.9 \mathrm{pc}$. The spectroscopic peculiarities of the SNR were demonstrated by Tuohy and Dopita (1983): spectra of individual knots showed strong lines of oxygen and neon, but nothing else. Velocity mapping indicated an overall expansion velocity of $\sim 6500 \mathrm{~km} \mathrm{~s}^{-1}$, perhaps distributed in a twisted ring morphology. These characteristics imply an expansion age for the remnant (assuming no deceleration) of 2150 years.

Objects with inferred large abundances of such elements as oxygen, neon, sulfur, etc., are thought to represent the explosions of massive (Population I) stars. Nuclear burning in the progenitor stars had progressed to or perhaps even beyond the oxygen-burning stage when the SN explosion took place. Other members of the class include N132D and 0540-69.3 in the LMC , Cas A and G292.0 + 1.8 in our Galaxy, and the powerful young SNR in the irregular galaxy NGC 4449. More recently, some knots dominated by oxygen emission have been identified in Puppis A (Winkler and Kirshner 1985), although this remnant is thought to be considerably older.

Some heavy elements such as carbon, silicon, and magnesium have no strong lines in the optical and their abundances are unconstrained by optical spectra alone. Also, so few lines are available in the optical that it is difficult to constrain models for the emission. Since the reddening to $1 \mathrm{E} 0102-7219$ is fairly small, we have used the IUE Observatory to obtain UV spectra of this remnant. New optical data have also been obtained, extending spectral coverage to 1 micron. These new data provide considerable insight into the physical conditions and possible emission mechanisms in this remnant. 


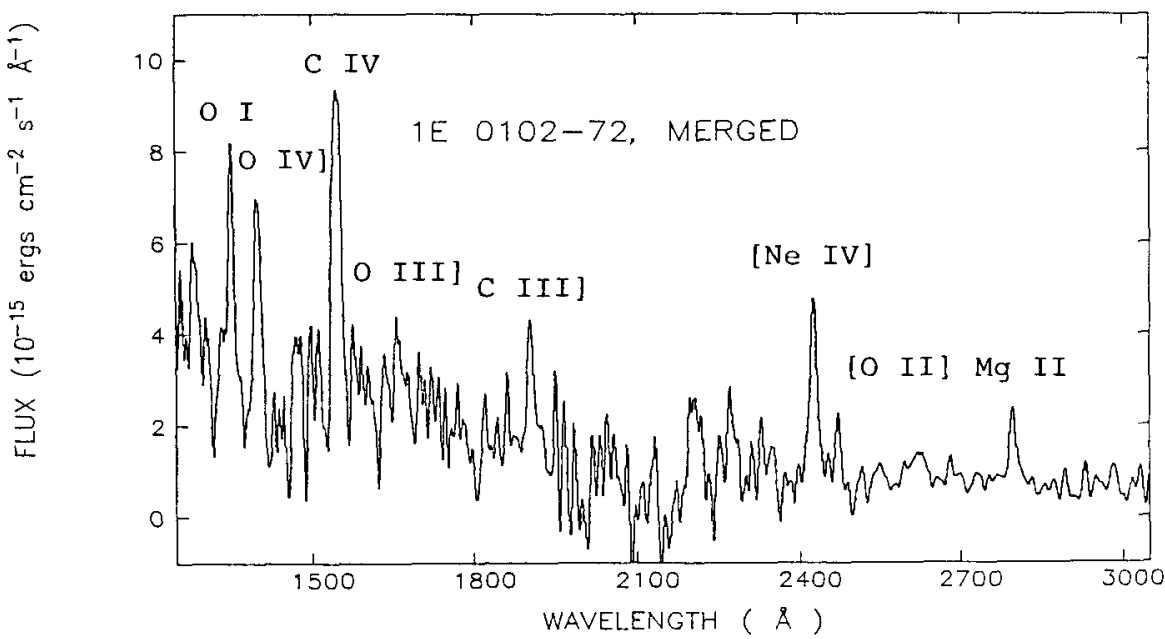

Figure 1: Merged SWP+LWP spectrum of 1E 0102-7219.

Table 1

Optical/UV Line Intensities for 1E 0102-7219

\begin{tabular}{lccc}
\hline \multicolumn{1}{c}{ Ion } & $\lambda$ & $F(\lambda 5007=100)$ & $\mathrm{I}(\lambda 5007=100)$ \\
\hline O I] & 1356 & 12.1 & 20.2 \\
Si IV/O IV] & 1400 & 19.6 & 31.5 \\
C IV & 1549 & 35.7 & 52.0 \\
O III] & 1664 & 6.0 & 8.4 \\
C III] & 1909 & 8.0 & 10.6 \\
{$[\mathrm{Ne} \mathrm{IV]}$} & 2425 & 15.2 & 18.3 \\
{$[\mathrm{O} \mathrm{II}]$} & 2470 & 4.9 & 5.8 \\
$\mathrm{Mg} \mathrm{II}$ & 2798 & 5.9 & 6.4 \\
{$[\mathrm{Ne} \mathrm{V}]$} & 3426 & 9.5 & 10.8 \\
{$[\mathrm{O} \mathrm{II}]$} & 3727 & 150 & 164 \\
{$[\mathrm{Ne} \mathrm{III}]$} & 3869 & 19.0 & 20.7 \\
{$[\mathrm{O} \mathrm{III}]$} & 4363 & 4.6 & 4.8 \\
{$[\mathrm{O} \mathrm{III}]$} & 5007 & 100 & 100 \\
{$[\mathrm{O} \mathrm{I}]$} & 6300 & 5.0 & 4.7 \\
{$[\mathrm{O} \mathrm{II}]$} & 7320,30 & 10.0 & 9.0 \\
O I & 7774 & 3.6 & 3.2 \\
\hline
\end{tabular}

Table Notes: 1) UV to optical scaling assumes a theoretical ratio of $\mathrm{I}(1356) / \mathrm{I}(7774)=5.9$. 2) Reddening correction assumes $\mathrm{E}(\mathrm{B}-\mathrm{V})=0.04$ (galactic) plus $\mathrm{E}(\mathrm{B}-\mathrm{V})=0.04$ (SMC).

\section{Observations}

For the IUE observations, we combined pairs of back-to-back ESA and US1 shifts to obtain long (14+ hour) SWP and LWP exposures of the SNR. The observations were scheduled to permit the long dimension of the large aperture to be roughly aligned along the bright southeastern rim of the [O III] ring visible in Tuohy and Dopita's (1983) photograph. Because of the long exposures, the background levels are fairly high, but emission lines from the SNR are clearly visible on the photowrites as diffuse patches of emission. 
We have extracted the spectra from the line-by-line data in order to optimize the signal-to-noise ratio of the resulting spectra. The merged SWP+LWP spectrum is shown in Figure 1 after removal of hits and reseau marks and after smoothing. The strongest line is $\mathrm{C}$ IV $\lambda 1550$, and $\mathrm{C} \mathrm{III]} \lambda 1909$ and the Si IV/O IV] blend at $\lambda 1400$ are also seen protruding from a noisy continuum. In the $L W P$ range, only three lines are clearly seen: $[\mathrm{Ne} I V] \lambda 2425$, [O II] $\lambda 2470$ and $\mathrm{Mg}$ II $\lambda 2800$. A surprising feature in this spectrum is the strong line at $\sim 1354 \AA$ which we identify as a recombination line of $O I$. Conspicuous by its near absence is the $O$ III] $\lambda 1664$ line, which was the only line detected in the UV spectrum of the SNR in NGC 4449 (Blair et al. 1984); interestingly, no carbon lines were seen in the NGC 4449 remnant.

Our optical data were obtained with long slit CCD spectrographs on the ESO $3.6 \mathrm{~m}$ telescope and the $4 \mathrm{~m}$ telescope at CTIO. In the 7-10000 $\AA$ region, several permitted O I transitions are seen, the strongest of which is at $7774 \AA$. The relative intensities of these lines indicate partially optically thin conditions in the recombining gas. Since the $7774 \AA$ line feeds the $1356 \AA$ line seen in the UV, the line intensities throughout the optical/UV region can be scaled. An abbreviated list of the most important lines is given in Table 1.

\section{Models}

Shocks are a natural explanation for the observed emission in that the ejecta encounter a 'reverse' shock when they overtake the decelerating high pressure shell of swept-up interstellar material. While most of the material passing through the reverse shock becomes so hot it emits at X-ray, rather than optical, wavelengths, the slower shocks in dense clumps of ejected gas can produce optical emission. Chevalier and Kirshner (1978) interpreted the Fast Moving Knots in Cas A as such shocks, and this picture is supported by the sudden appearance and disappearance of many of these knots ( $c f$. van den Bergh and Kamper 1983).

However, only a few shock models have been calculated for abundances appropriate to oxygen-rich remnants. Itoh (1981) constructed models of radiative shocks in pure oxygen and extended them to include O I recombination (Itoh 1987). Dopita, Binette and Tuohy (1984; hereafter DBT) computed models for a mixture of $\mathrm{C}, \mathrm{O}$ and O-burning products expected from a $25 \mathrm{M}_{\odot}$ precursor. We have used a modified version of the radiative shock code described in Cox and Raymond (1985) to compute a few models with higher or lower shock velocity than those of Itoh or DBT, models with different abundance sets, and models which include the observed X-ray emission of $1 \mathrm{E}$ 0102-7219. All these models assumed instant electron-ion equilibration. Aside from a general confirmation of the published models, we find that the X-ray emission alone can completely ionize any gas less dense than about $1 \mathrm{~cm}^{-3}$, so that even precursors of very slow shocks can be ionized.

Problems arise in attempting to match the observed high ionization species $\mathrm{Ne}$ IV and $\mathrm{Ne} \mathrm{V}$, and in matching the ratios of UV lines to the optical. Models which produce $[\mathrm{O} \mathrm{II}] /[\mathrm{O}$ III] and [O I]/[O III] comparable to the observed values predict no emission at all in [Ne IV] or $[\mathrm{Ne} \mathrm{V}]$, because the gas never reaches high ionization states. Models which collisionally ionize oxygen to $\mathrm{O}^{++}$have high enough temperatures to excite strong UV emission, but the observed $\mathrm{UV}$ lines are rather weak. The $[\mathrm{Ne} \mathrm{IV}]$ and $[\mathrm{Ne} \mathrm{V}]$ problem could be explained by assuming a wide range of shock velocities within the observed volume, but any shocks which produce these lines would give a C IV $\lambda 1550 / \mathrm{C} \mathrm{III]} \lambda 1909$ ratio far larger than observed. Also, this would not alleviate the problem with the O III UV to optical line ratio.

The X-ray luminosity of $1 \mathrm{E}$ 0102-7219 is $1.5 \times 10^{37} \mathrm{ergs}^{-1}$, so X-ray photoionization may be considerable. Since detailed measurements of the X-ray spectrum are not available, we have used the X-ray emission code of Raymond and Smith (1977) with updated atomic rates to generate model $X$-ray spectra covering the $0-2 \mathrm{KeV}$ range at $4 \mathrm{eV}$ resolution. Either the DBT abundance set or a pure oxygen gas was assumed. Temperatures of $2.5 \times 10^{6}$ and 6 
$\times 10^{6} \mathrm{~K}$ were chosen. The spectra were then scaled to match the observed flux from the remnant. Each X-ray spectrum was used to compute the ionization state and heating rate of gas at various densities, and a temperature at which heating balances cooling was found.

From inspection of the models, photoionized gas with a range of densities emits strongly in $[\mathrm{O}$ III] $]$. It can produce enough $[\mathrm{O}$ I] $\lambda 6300$ and $\mathrm{O}$ I $\lambda 7774 \mathrm{emission}$ in some of the denser regions, and it naturally produces the observed modest UV to optical line ratios. There are two difficulties, however. First, the predicted $[\mathrm{O}$ II $] \lambda 3727 /[\mathrm{O}$ III $] \lambda 5007$ intensity ratio is less than 0.3 in all cases, while the observed ratio varies between about 1.3 and 2 . Second, the [O III] emission is dominated by 10-15000 K gas, while the temperature-sensitive [O III] $4363 / 5007$ ratio indicates a temperature of $25000 \mathrm{~K}$. The O III line ratio might be explainable by a recombination contribution to the $\lambda 4363$ line. The weakness of the predicted $[\mathrm{O}$ II] seems to be a more fundamental problem, reflecting the fact that at densities high enough that $\mathrm{O}^{+}$is more abundant than $\mathrm{O}^{++}$, the temperatures are too low to overcome the $3.3 \mathrm{eV}$ excitation potential of the $\lambda 3727$ lines.

The fault probably lies in the assumption of ionization equilibrium. While the heating and cooling time scales are a few years, the ionization time scale is $\sim 2000$ years, which is roughly the expansion age of the remnant. Thus the gas may be less highly ionized than predicted by the equilibrium models for a given density, and it will probably be warmer, since the power provided by X-ray heating is shared among fewer electrons. A timedependent model would probably give an $[\mathrm{O} \mathrm{II}] /[\mathrm{O} \mathrm{III}]$ ratio in agreement with that observed. It could probably also account for the high ionization stages-- $\mathrm{C} \mathrm{V}, \mathrm{Ne} \mathrm{TV}$ and $\mathrm{Ne}$ V--because only two Auger ionizations are required.

Although we have been unable to determine a unique model, the combined optical/UV data shed light on several aspects of this remnant. Many of the general characteristics of the observed emission can be accounted for with the X-ray photoionization models, but shocks may also play an important role. Oxygen is the dominant element, but substantial amounts of neon, carbon and magnesium are also present. The relative abundances are crudely like those expected from a star of about $20 \mathrm{M}_{\odot}$. Further refinements in the relative abundances should permit the mass of the precursor star to be determined more accurately.

\section{References}

Blair, W.P., Raymond, J.C., Fesen, R.A., and Gull, T.R. 1984, Ap. J., 279, 708.

Chevalier, R.A. and Kirshner, R.P. 1978, Ap. J., 219, 931.

Cox, D.P. and Raymond, J.C. 1985, Ap. J., 288, 651.

Dopita, M.A., Binette, L., and Tuohy, I.R. 1984, Ap. J., 282, 142.

Dopita, M.A., Tuohy, I.R., and Mathewson, D.S. 1981, Ap. J. (Letters), 248, L105.

Itoh, H. 1981, Pub. Astr. Soc. Japan, 33, 1.

--1987, Pub. Astr. Soc. Japan, , in press.

Raymond, J.C. and Smith, B.W. 1977, Ap. J. Suppl., 35, 419.

Seward, F.D. and Mitchell, M. 1981, Ap. J., 243, 736.

Tuohy, I.R. and Dopita, M.A. 1983, Ap. J. (Letters), 268, L11.

van den Bergh, S. and Kamper, K.W. 1983, Ap. J., 268, 129.

Winkler, P.F. and Kirshner, R.P. 1985, Ap. J., 299, 981. 